\title{
Frequent witnessing to interparental conflict and low parental availability for leisure-time is associated with dysfunctional voiding in primary school children
}

\author{
Ahmet Cihan ${ }^{1}$ and Adnan Ünalan ${ }^{2}$ \\ ${ }^{1}$ Affiliation not available \\ ${ }^{2}$ Niğgde Ömer Halisdemir University
}

October 18, 2020

\begin{abstract}
Aim: Aspects of parenting is an under-investigated topic among children with voiding complaints. We aimed to investigate the association between voiding complaints among children and two specific parenting related behaviors: 1) parental availability during the child's leisure time and 2) frequency of witnessing interparental conflict. Material and Method: Children aged between 6 and 11 years with their parents were the participants. Two survey forms and informed consent form were sent to potential participants via children's teacher. Voiding dysfunction complaints were evaluated with the Dysfunctional Voiding and Incontinence Symptom Scale (DVISS). Results: Data analyses were performed with 1101 eligible participants. Mean duration of parental availability during the child's leisure time was below 2 hours per day. Nearly one out of every four children witnessed interparental conflict at least once a month. Age, educational level of the mother, parental availability for the child's leisure time, and frequency of witnessing parental conflicts were found to be significantly associated with DVISS ratings. Adjusting age, socioeconomic level, and educational level of the parents and later adjusting parental availability during the child's leisure time did not change the association of frequency of witnessing interparental conflict with DVISS rates. Increase in the age, educational level of the mother, and parental availability during the child's leisure time were correlated with better scores on DVISS while increase in the frequency of witnessing interparental conflict strongly correlated with worse scores. Conclusion: Exposing children to interparental conflicts and shorter togetherness with their leisure time is associated with voiding complaints.
\end{abstract}

\section{Hosted file}

Main Text.odt available at https://authorea.com/users/353507/articles/487342-frequentwitnessing-to-interparental-conflict-and-low-parental-availability-for-leisure-time-isassociated-with-dysfunctional-voiding-in-primary-school-children 

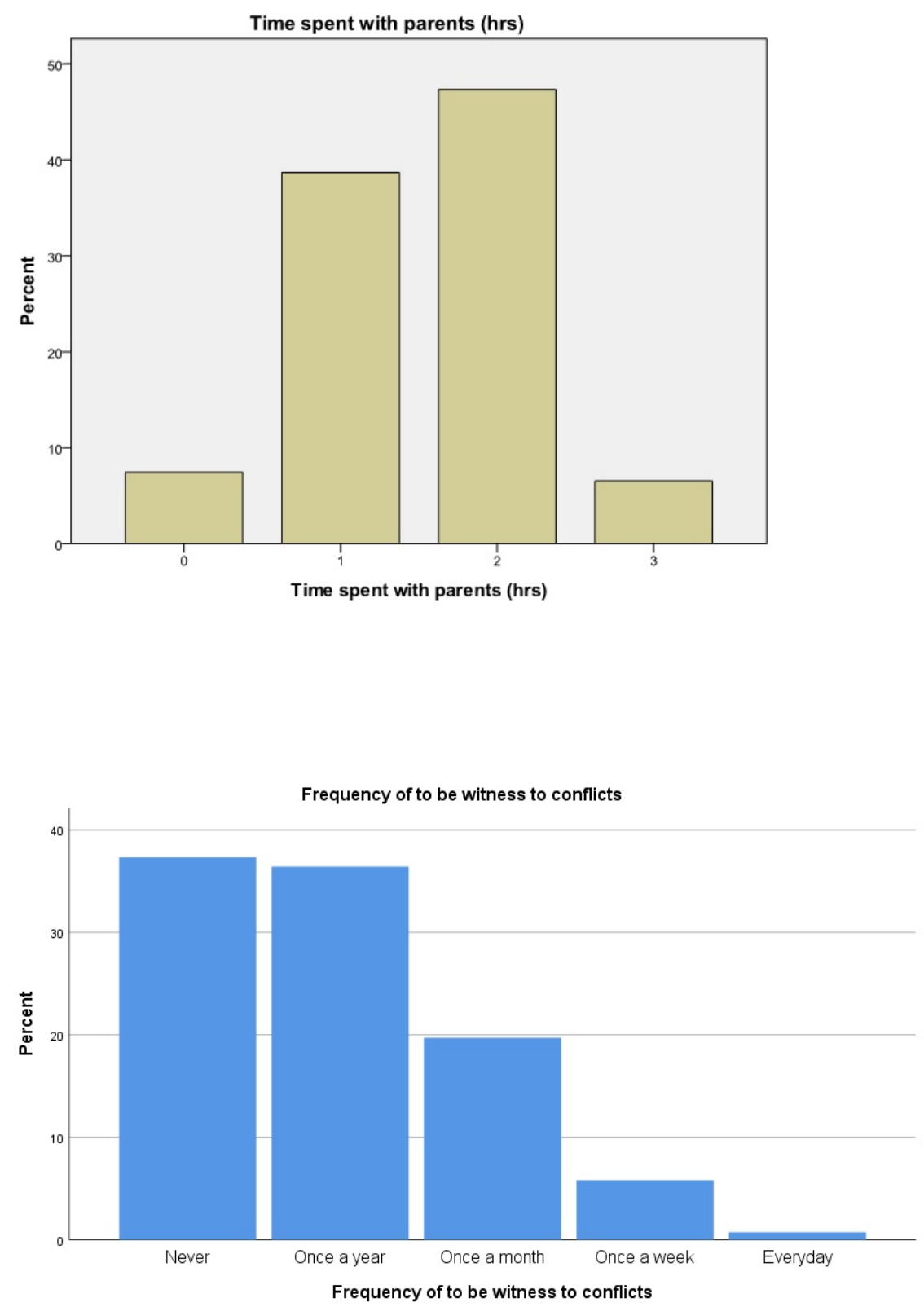